\title{
LETTERS
}

\section{Bringing family medicine into the modern age}

As a family physician who is a millennial, I found Wendy Glauser's CMAJ News arti$\mathrm{cle}^{1}$ to ring true. What many physicians, and certainly funders, across Canada have yet to realize is that the way millennials communicate with friends, family and businesses on a daily basis has changed dramatically - we text. The essential aspect of this medium of communication is that it is asynchronous.

Asynchronous messaging (or as some vendors of electronic medical records call it, "secure text") has been successful in some longitudinal primary care clinic systems in the United States (e.g., Sherpaa), and has provided equivalent continuity of care for patients while increasing patient convenience and creating system efficiencies and cost savings. Some clinics that have embraced secure asynchronous messaging have seen $50 \%$ or more of "visits" occurring virtually and asynchronously, while providing equivalent care and continuity (www.youtube.com/ watch?v=93vozgHfTB8).

Unfortunately, in Canada, no funding is currently available in most provinces for use of this technology, and vendors of electronic medical records seem loathe to build out this capability where it does not directly increase their revenue.

We need to push policy-makers to help bring family medicine into the modern age. As many of my patients have said to me, "I can text my hair dresser and my dentist ... why can't I text you?"

\section{Brady Bouchard MBBS}

Family physician and emergency physician, Department of Academic Family Medicine, University of Saskatchewan, North Battleford, Sask.

- Cite as: CMAJ 2019 March 25;191:E343. doi: $10.1503 / \mathrm{cmaj} .71522$

\section{Reference}

1. Glauser W. Primary care system outdated and inconvenient for many millennials. CMAJ 2018;190:E1430-1.

Competing interests: None declared. 